\title{
“Tuneo” de proteínas: herramientas para modificarlas
}

\author{
José Fernando García Guevara
}

\section{Resumen}

Las proteínas son componentes moleculares esenciales que se encargan de realizar gran parte de los procesos que nos mantienen vivos. Las proteínas que existen naturalmente son muy diversas y cada una de ellas cumple una tarea. A continuación, se presenta la disciplina que se encarga de su "tuneo", la ingeniería de proteínas, que estudia cómo funcionan las proteínas y utiliza este conocimiento para desarrollar herramientas con las que se puedan realizar modificaciones específicas en ellas. Asimismo, se exponen algunas de las herramientas en dicha disciplina, junto a casos particulares donde se han empleado. Al final, se propone cómo combinar dichas herramientas para mejorar los resultados de las modificaciones deseadas.

Palabras clave: proteínas, enzimas, ingeniería de proteínas.

\section{"TUNING" OF PROTEINS: TOOLS TO MODIFY THEM}

\begin{abstract}
Proteins are essential molecular components of life; they have a role in almost every of its processes. Proteins in nature are diverse and each one of them performs a particular task. In this article we introduce the discipline in charge of "tuning" them, protein engineering, which studies how proteins work and uses this knowledge to develop tools to make custom modifications on them. Also, we present some of the tools employed in it and particular cases where they have been used. Finally, we propose how can we combine such tools to improve the results of desired modifications.
\end{abstract}

Keywords: protein, enzymes, protein engineering. 
Estudiante del doctorado en Ciencias Bioquímicas en el Departamento de Ingeniería Celular y Biocatálisis del Instituto de Biotecnología, Universidad Nacional Autónoma de México (UNAM). Licenciado en Ciencias Genómicas y maestro en Ciencias Bioquímicas por la UNAM.

\section{Introducción}

Todos hemos visto alguna vez (por lo menos en películas) un automóvil "tuneado", es decir, un carro con modificaciones sobre su diseño de fábrica que cambian sus características y le dan una nueva personalidad. Estas alteraciones permiten tener autos más ruidosos, más rápidos y más furiosos. Usando una caja de herramientas, los mecánicos de algunos talleres especializados averiguan cómo funciona cada coche y luego cambian o agregan las piezas necesarias para cumplir las especificaciones del cliente. En nuestro grupo de trabajo, de manera semejante, usando otras herramientas, tratamos de "tunear" las proteínas y modificar sus características de acuerdo a nuestras necesidades.

Podemos imaginar a las proteínas como máquinas diminutas encargadas de realizar los procesos que nos mantienen vivos. Existen veinte piezas distintas que componen a las proteínas y son llamadas aminoácidos. Un aminoácido es una molécula formada por tres partes: una parte amino, una parte carboxilo y una cadena lateral. La parte amino y carboxilo son el elemento en común en los veinte tipos de aminoácidos, mientras la cadena lateral distingue a cada uno de ellos y les brinda propiedades físicas y químicas distintivas. La parte amino de un aminoácido va enlazada con la parte carboxilo de otro aminoácido, hasta formar una larga cadena. Las cadenas laterales quedan libres y están ordenadas en una secuencia particular, ésta es la secuencia de la proteína (figura 1). Las propiedades físicas y químicas de las cadenas laterales, ordenadas en una secuencia determinada, provocan el plegamiento de la proteína para que adquiera una estructura particular, con la que es capaz de realizar su función. Al igual que las piezas en un motor están colocadas con precisión y forman una estructura compleja, las piezas de una proteína quedan orientadas en forma precisa para funcionar (figura 2). 
a)

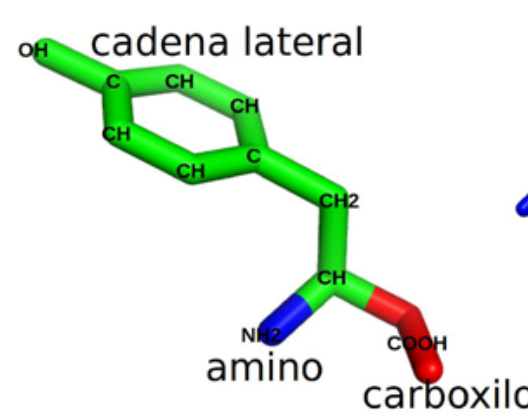

b)

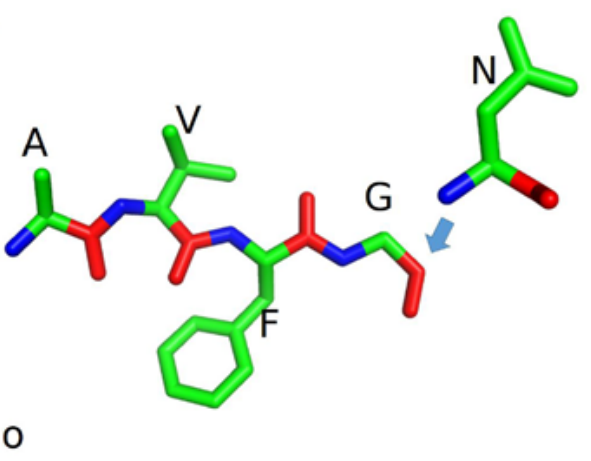

Figura 1. Ensamblaje y secuencia de las proteínas.

a) Ejemplo de la estructura de una de las veinte piezas que forman parte de las proteínas, se trata del aminoácido llamado tirosina. En azul se muestra la parte amino; en rojo, la parte carboxilo, y en verde, la cadena lateral.

b) La parte carboxilo de un aminoácido (en color rojo) se enlaza a la parte amino de otro aminoácido (en color azul), formando un enlace peptídico. Los aminoácidos se van uniendo uno al lado de otro para formar una cadena con una secuencia particular.; en el ejemplo mostramos sólo un fragmento de la secuencia en una proteína. Este fragmento está constituido por cinco aminoácidos distintos (alanina, valina, fenilalanina, glicina y asparangina), que forman la secuencia AVFGN — cada letra en la secuencia es un código que corresponde al nombre del aminoácido-.

Figura 2. De la secuencia a la estructura de una proteína. Arriba se muestra la secuencia de aminoácidos de la proteína precursora de la insulina y abajo su estructura. Después de que se sintetiza la cadena de aminoácidos, ésta adquiere una estructura definida; la cual, después de una ligera modificación se convierte en insulina, hormona de naturaleza proteica que regula el nivel de azúcar en la sangre.

\section{Secuencia}

FVNQHLCGSDLVEALYLVCGERGFFYTDKGIVEQCCTSICSLYQLENYCN
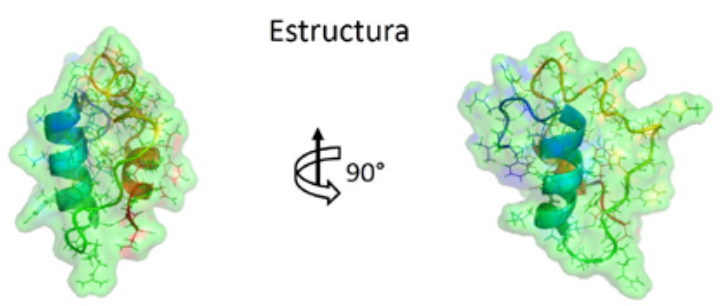

Las proteínas cumplen una amplia variedad de papeles: pueden ser estructurales como el colágeno en nuestra piel y huesos, o transportar oxígeno como la hemoglobina en la sangre, o también pueden acelerar reacciones químicas (ver figura 3). Las proteínas hacen (literalmente) posible nuestra vida, aunque cuando se descomponen también pueden arruinarla: ejemplos dramáticos de ello lo representan más de un centenar de enfermedades de origen genético. Un caso es la fenilcetonuria, una enfermedad hereditaria que provoca que el aminoácido fenilalanina, proveniente de los alimentos, se acumule en el cuerpo, lo que a largo plazo daña al sistema nervioso central y al cerebro. La enzima fenilalanina hidroxilasa controla los niveles de fenilalanina, pero variaciones genéticas provocan ligeros cambios en la secuencia de aminoácidos, dejándola inactiva y causando así la enfermedad (Blau, 2016). 
Figura 3. Ejemplos de algunas proteínas. Se muestra la estructura de algunas proteínas presentes en el cuerpo humano y que participan en distintos procesos. Arriba a la izquierda, la estructura del colágeno; esta proteína forma fibras que constituyen la mayor parte de la estructura de la piel y los huesos. Arriba a la derecha, la estructura de la proteína supresora de tumores p53, que se une a

la cadena de ADN para realizar su función; con frecuencia se ha encontrado que esta proteína ha mutado en algunos tipos de cáncer. Abajo a la izquierda se muestra la estructura de la lactasa, que ayuda a digerir la lactosa; cuando no se sintetiza en cantidades suficientes produce intolerancia a la lactosa. Abajo a la derecha, la estructura de la hemoglobina, presente en las células de la sangre y encargada de unir y transportar el oxígeno que requieren las demás células en nuestro cuerpo; algunas mutaciones en esta proteína causan una enfermedad conocida como anemia falciforme.
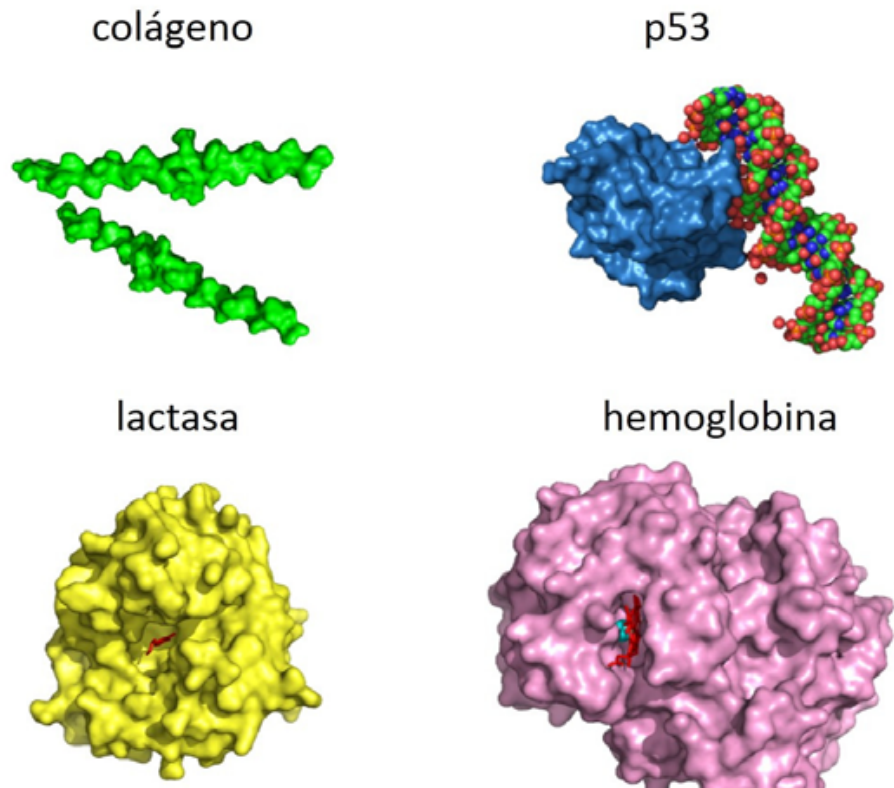

hemoglobina

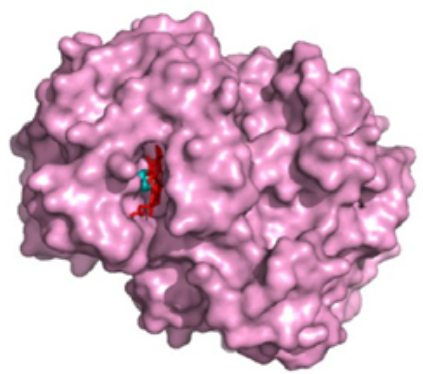

Las proteínas que aceleran reacciones químicas se conocen como enzimas. Las enzimas actúan sobre moléculas llamadas sustratos, y las transforman químicamente en moléculas distintas denominadas productos. La actividad de una enzima se mide como la velocidad a la que transforma un sustrato en producto. La velocidad en los autos se mide como la distancia que recorre por unidad de tiempo, en las enzimas, se mide como el número de moléculas de sustrato que transforma en producto por unidad de tiempo. El sitio activo de una enzima es una zona de la enzima donde la orientación precisa de las cadenas laterales permite reconocer al sustrato y acelerar la reacción química.

\section{Herramientas en la ingeniería de proteínas}

La secuencia de una proteína determina sus propiedades, por ejemplo, qué tan rápida es o a qué temperatura puede trabajar; esto significa que si modificamos la secuencia de una proteína, entonces, podemos modificar sus propiedades. Pero el problema que enfrentamos es saber cuáles son los cambios que necesitamos para "tunear" a una proteína de acuerdo a nuestras especificaciones; por ejemplo, que sean más veloces, usen nuevos sustratos o resistan temperaturas más altas. La ingeniería de proteínas es la disciplina que se encarga de resolver este problema y para lograrlo estudia la relación que existe entre la secuencia, la estructura y la función de las proteínas; además, desarrolla y emplea las herramientas o estrategias que nos permitan resolverlo.

Retomemos el ejemplo de un motor e imaginemos que queremos modificarlo para que ahora tenga mayor potencia. Lo primero que debemos saber es cómo funciona, cómo son las piezas que lo componen y cómo es que 
cuando se ensamblan generan movimiento. Este conocimiento permite saber qué herramientas usar y qué cambios hacerle al motor para lograr nuestra meta. Algo semejante pasa con la ingeniería de proteínas, primero debemos adquirir el conocimiento acerca de cómo funcionan las proteínas, y luego lo aplicamos en el desarrollo de herramientas y estrategias para "tunearlas".

Una de las herramientas desarrolladas es el diseño por computadora: un programa especializado que modela las interacciones a nivel atómico que ocurren en la proteína y predice los cambios adecuados para que una proteína cambie su función original. Desafortunadamente, nuestro conocimiento acerca de la relación entre la secuencia, la estructura y la función de las proteínas todavía no es completo, por lo que los modelos usados en el diseño de proteínas por computadora no siempre son acertados. Por lo anterior, es necesario usar otras herramientas. Entre las que dan mejores resultados se encuentra la evolución dirigida.

Las proteínas han sido optimizadas a través de un proceso conocido como evolución: naturalmente algunas proteínas sufren cambios al azar, cuando esos cambios presentan alguna ventaja para la supervivencia y la reproducción del organismo, entonces, ese cambio permanece y se transmite a generaciones posteriores, es decir, se selecciona (Valero y Jardón, 2006). El proceso de evolución es algo así como prueba y error, primero se realizan cambios al azar y si son útiles se quedan, si no, se van. Con la evolución dirigida tratamos de emular el proceso de evolución. Primero generamos muchas variantes sin saber qué efecto van a tener, y después usamos un sistema de selección que nos permita quedarnos con las variantes que cumplan con nuestros criterios. Un punto crucial de la estrategia es contar con un sistema de selección eficiente, que nos permita probar el mayor número de variantes y que elija con precisión la característica deseada. Con un buen sistema de selección, dicha herramienta es grandiosa. Desafortunadamente no siempre contamos con la mejor forma de identificar las mejores proteínas, es entonces cuando usar esta herramienta se vuelve una tarea ardua y costosa.

La ingeniería de proteínas ha logrado avances significativos en las herramientas desarrolladas, pero el camino que falta recorrer es largo. En nuestro grupo, propusimos tomar una herramienta ya existente, conocida como ingeniería de consensos, y darle una nueva aplicación como herramienta auxiliar al diseño por computadora. La ingeniería de consensos consiste en tomar un grupo de secuencias de aminoácidos de proteínas semejantes y con ellas construir una nueva secuencia, usando aquellos que se presentan con mayor frecuencia (ver figura 4). Esta estrategia se usa para obtener proteínas que puedan trabajar a temperaturas más altas de las que normalmente lo hacen, pero algunas observaciones sugieren que se podría usar para aumentar la actividad con nuevas moléculas (Aerts, Verhaeghe, Joosten, Vriend y Soetaert, 2013; Loening, Fenn, Wu y Gambhir, 2006). La ingeniería de consensos aprovecha los recursos y la diversidad que nos ofrece la naturaleza; las mutaciones que propone esta herramienta ya han probado ser exitosas, lo cual disminuye la probabilidad de 
Figura 4. Ingeniería de consensos.

En la ingeniería de consensos se construye una nueva secuencia basada en el "voto" de la mayoría. En el ejemplo, primero se reúne la secuencia de cinco proteínas similares y se alinean. Luego se selecciona el aminoácido más frecuente en cada posición del alineamiento, y es así como al final se termina con una secuencia consenso.

En el ejemplo resulta que la secuencia consenso es idéntica a la secuencia 1, es decir, no hay mutaciones consenso.

Figura 5. La enzima fitasa. Se muestra la estructura de la enzima fitasa (en color verde), la cual se une al ácido fítico en su sitio activo (en color blanco). La fitasa libera los grupos fosfato del ácido fítico, al realizar un corte enzimático (conocido como hidrólisis) en los enlaces señalados con flechas rojas. introducir cambios que perjudiquen nuestro diseño.

$\begin{array}{lll}\text { secuencia } & 1 & \text { METYAVFGNPIAHSKSPFIHQQFAQQ } \\ \text { secuencia } & 2 & \text { MEKYAVFGNPIAHSKSPFIHQQFAQQ } \\ \text { secuencia } & 3 & \text { METYAVFGNPIAHSKSPSIHQLFARQ } \\ \text { secuencia } & 4 & \text { METYAVFGNPVSHSKSPFIHQQFAQQ } \\ \text { secuencia } & \text { MHTFAVFGNPINHSKSPRIHQLFAEQ } \\ \text { consenso } & & \text { METYAVFGNPIAHSKSPFIHQQFAQQ }\end{array}$

Las primeras secuencias consenso que se construyeron fueron en proteínas denominadas inmunoglobulinas. Estas proteínas forman parte del sistema inmune y se encargan de detectar y neutralizar elementos extraños. Se observó que las inmunoglobulinas consenso resistían temperaturas más altas (Steipe, Schiller, Pluckthun y Steinbacher, 1994). Sin embargo, dentro de las funciones de esas proteínas no está la de acelerar reacciones químicas. Viendo las propiedades producidas por la ingeniería de consensos, un grupo de investigadores de la compañía farmacéutica Roche propuso aplicar la estrategia a una proteína que sí acelera reacciones químicas. Eligieron a la proteína fitasa, la cual pensaban usar para suplementar el alimento de aves de corral. El fósforo es un elemento importante en la dieta de aves de corral, por eso su alimento se adiciona con fosfato inorgánico, lo malo es que esto provoca un daño ambiental. El alimento para aves de corral contiene fósforo en forma de ácido fítico, el cual proviene de las plantas con las que se prepara el alimento; pero las aves no lo pueden asimilar porque su sistema digestivo carece de fitasas, las enzimas que liberan el fósforo del ácido fítico (ver figura 5). La solución propuesta: agregar fitasas al alimento para liberar el fósforo del ácido fítico. Sin embargo, el proceso de preparación del alimento requiere temperaturas superiores de las que resisten las fitasas naturales. Al aplicar la ingeniería de consensos, usando las secuencias de las fitasas conocidas crearon nuevas secuencias de fitasas que toleran temperaturas más altas, sin perder su capacidad para liberar el fosfato del ácido fítico (Lehmann et al., 2000).

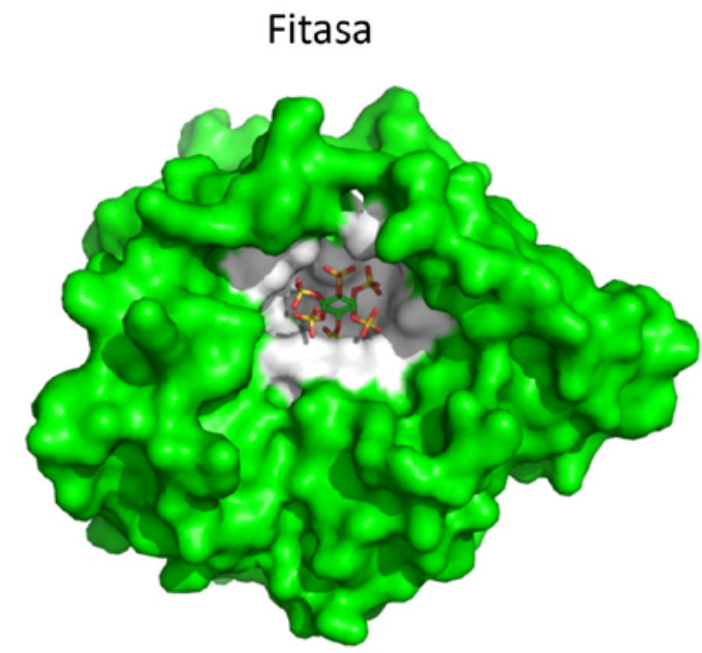

Ácido fítico 
La ingeniería de consensos siempre ha tenido en la mira obtener proteínas capaces de trabajar a temperaturas más elevadas. Pero algo que a nosotros nos llamó la atención es que en algunos trabajos probaron la actividad de las proteínas consenso con nuevos sustratos, y encontraron mayor actividad sobre los nuevos sustratos comparados con la proteína original (Aerts et al., 2013; Loening et al., 2006). Estas observaciones nos llevaron a pensar que podemos aprovechar la ingeniería de consensos para mejorar la actividad con nuevos sustratos y poder complementar otras herramientas como el diseño por computadora.

\section{Un pedido frecuente: intercambio de actividad de NADP a NAD}

Las reacciones químicas dentro de un organismo se parecen a una línea de ensamblaje: una molécula inicial se va modificando paso a paso hasta llegar a un producto final, y cada paso lo realiza una enzima específica. Algunas de esas reacciones involucran la transferencia de electrones (reacciones de oxidación-reducción) y las enzimas encargadas de acelerar dichas reacciones, por lo general, necesitan de otras moléculas. Así, las coenzimas son moléculas accesorias de algunas enzimas, pero indispensables para su actividad; algo así como las baterías en un control remoto, no son parte del control, pero éste las requiere para funcionar. De esta manera, el sitio activo de una proteína reconoce a su coenzima junto con el sustrato y los modifica químicamente durante la reacción. Dos de las coenzimas más comunes en las células son la Nicotinamida Adenina Dinucleótido (NAD) y la Nicotinamida Adenina Dinucleótido Fosfato (NADP). Éstas moléculas son muy parecidas, pero en la naturaleza tienen papeles diferentes. NAD se usa principalmente en reacciones de oxidación, mientras que NADP usualmente está presente en reacciones de biosíntesis. Por ejemplo, cuando comemos azúcar la quemamos (oxidamos) para liberar energía y luego usamos esa energía para realizar nuestras actividades; algunas de las enzimas involucradas en ese proceso usan NAD. Si comemos mucha azúcar puede que liberemos energía de sobra, la cual se puede almacenar en forma de grasa corporal: para su síntesis participan enzimas que usan NADP.

Un pedido frecuente en el "tuneo" de proteínas es modificar a las proteínas que naturalmente usan NADP para que ahora usen NAD. NAD y NADP poseen propiedades similares, pero en ocasiones usar NAD presenta ventajas sobre NADP, pues el primero es más abundante dentro de la célula y en las condiciones adecuadas puede ayudar a mejorar la producción de algunos compuestos de interés comercial. Podríamos comparar este problema con intercambiar el sistema de combustible en un automóvil, digamos que en lugar de que consuma gasolina, como en general funciona un auto de fábrica, ahora queremos que consuma etanol o gas natural. 


\section{Tamiflu, influenza y shikimato deshidrogenasa}

La proteína con la que trabajamos en nuestro laboratorio se llama shikimato deshidrogenasa; su nombre refleja la actividad que realiza: producir shikimato (producto de esta reacción) a partir de 3-dehidroshikimato (sustrato de la reacción, ver figura 6). El shikimato es un compuesto de interés en la industria farmacéutica, en especial porque es la materia prima para producir un fármaco usado para combatir la influenza, llamado oseltamivir-fosfato, mejor conocido como Tamiflu de Roche. En el año 2009, la compañía farmacéutica Roche reportó que consume alrededor de 500 toneladas de shikimato para la producción anual de Tamiflu; aun así, esa cantidad no sería suficiente en el caso de una pandemia de influenza, ya que se estimó que para cubrir un caso semejante se necesitarían cerca de 39 mil toneladas de shikimato, esto es ocho veces la producción anual (Martínez, Bolívar y Escalante, 2015; Scheiwiller y Hirschi, 2010).

Figura 6. La enzima shikimato deshidrogenasa.

a) La enzima shikimato deshidrogenasa de E. coli usa al 3-deshidroshikimato como sustrato y lo convierte en shikimato. Para llevar a

cabo la reacción, la enzima usa NADP como coenzima, tomándolo en forma de NADPH y convirtiéndolo en NADP+.

b) En color verde se muestra la estructura completa de la enzima shikimato deshidrogenasa. La coenzima (en color amarillo) y el sustrato (en color azul) se unen al sitio activo (en color rojo) de la enzima para comenzar la reacción química.

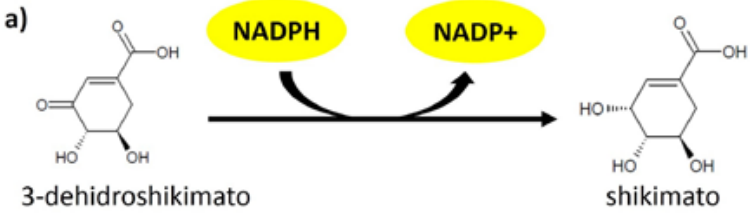

b)

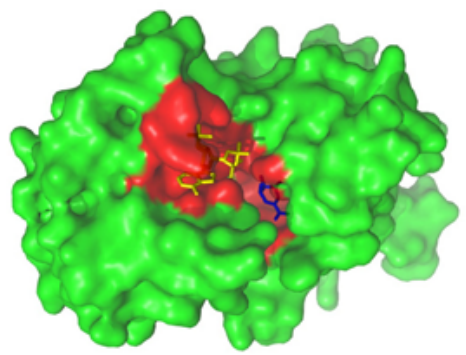

La fuente principal del shikimato es el fruto del anís estrella chino. La compañía Roche usa cerca del $90 \%$ de la cosecha total en el mundo. Extraerlo es costoso y poco eficiente: la planta requiere crecer durante seis años antes de la cosecha, la cual se debe realizar en septiembre y octubre. Esto dificulta cubrir la demanda creciente del fármaco, sobre todo después de los brotes recientes de influenza porcina y aviar (Kogure, Kubota, Suda, Hiraga y Inui, 2016).

Para realizar su función, la shikimato deshidrogenasa necesita NADP que, como comentamos arriba, es más escasa que NAD. De ahí que a nosotros nos interesó obtener una shikimato deshidrogenasa que pueda utilizar NAD y deje de usar NADP. Con la visión de que una enzima así pueda contribuir a las estrategias para producir shikimato, usando bacterias en lugar de cultivar plantas.

El objetivo de nuestro proyecto fue realizar un cambio de coenzimas en la enzima shikimato deshidrogenasa, para que prefiriera NAD en lugar de NADP. La enzima shikimato deshidrogenasa que seleccionamos proviene de una bacteria 
Figura 7. Intercambio de cofactores en la enzima shikimato deshidrogenasa de

E. coli.

El esquema muestra los pasos que se siguieron para probar la ingeniería de consensos como herramienta auxiliar para mejorar la actividad en la proteína diseñada por computadora. Las mutaciones consenso cercanas al sitio de unión son las que nos ayudan a aumentar la actividad sobre la proteína rediseñada.
Ilamada Escherichia coli, la cual habita en el colon humano y que ya se ha usado antes para producir shikimato (Martínez et al., 2015; Rodríguez et al., 2014).

Primero empleamos el diseño por computadora. Usamos un programa para el rediseño de enzimas conocido como Rosetta y desarrollado en la universidad de Washington. El programa recibe un modelo de la estructura de la enzima original junto a la nueva coenzima, en este caso NAD, y busca nuevas secuencias que mejoren la interacción con la coenzima. El resultado del programa es una nueva secuencia, la cual (según el programa) debería mejorar la actividad con la nueva coenzima. Después, introdujimos los cambios propuestos por el programa en la enzima shikimato deshidrogenasa y probamos su actividad. Sólo logramos que la proteína aumentara 50\% su actividad con NAD, pero disminuimos la actividad con NADP 1000 veces. La proteína ya prefería NAD, sin embargo, aún tenía baja actividad.

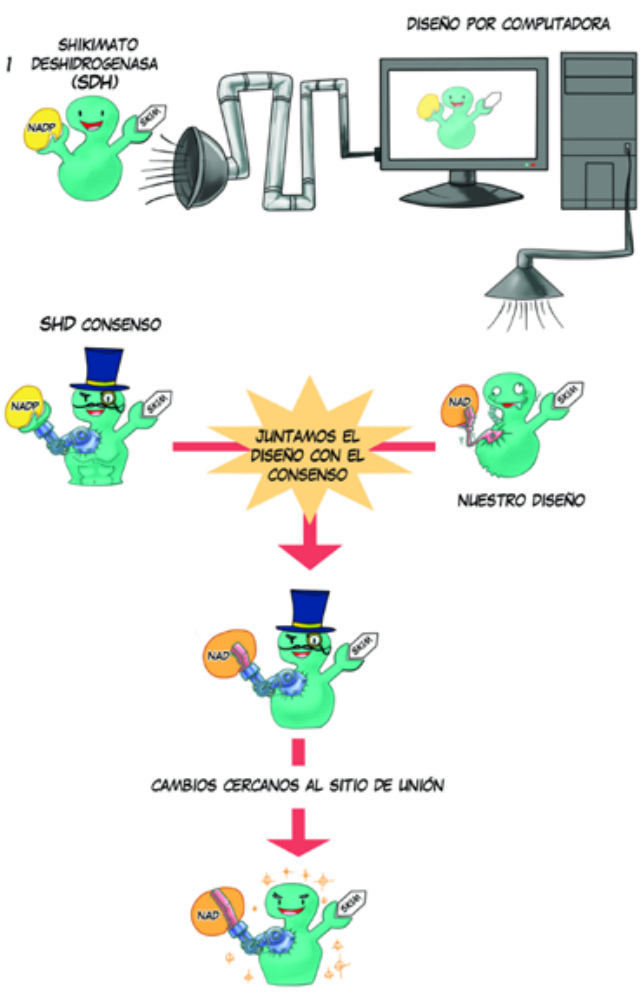

Por otro lado, construimos una secuencia consenso de la enzima shikimato deshidrogenasa usando la secuencia de E. coli y otras cincuenta secuencias de shikimato deshidrogenasa, provenientes de otras especies de bacterias. La secuencia consenso presentó 15 mutaciones comparada con la secuencia proveniente de E. coli; a estos 15 cambios los llamamos mutaciones consenso. Construimos la enzima consenso y vimos que conservaba su actividad con NADP. Observamos también actividad con NAD, no tanta como con NADP, pero sí mayor que la actividad con NAD de la enzima original de E. coli.

Por último, nos preguntamos si podíamos aprovechar la ingeniería de consensos para mejorar el diseño por computadora. Para averiguarlo añadimos las mutaciones consenso sobre la secuencia de la proteína que obtuvimos con el diseño por computadora. Usando los cambios propuestos por la ingeniería de consensos pudimos aumentar la actividad con NAD otras siete veces. También nos dimos cuenta de que las mutaciones consenso cercanas al sitio activo fueron las que provocaron este aumento en la actividad. Usando ambas herramientas para el diseño de proteínas (diseño por computadora e ingeniería de consensos) pudimos transformar a la shikimato deshidrogenasa (García-Guevara, Bravo, Martínez y 
Segovia, 2017), generando una proteína con "nueva personalidad" (ver figura 7). A pesar de este éxito, aún falta probar si podemos usar nuestra proteína modificada en la producción de grandes cantidades de shikimato.

\section{Conclusiones}

Así como un desarmador es diferente de una llave de tuercas, las herramientas que empleamos en la ingeniería de proteínas también son distintas entre sí, se complementan unas con otras y se aplican dependiendo del lugar donde nos encontremos y el destino al que vayamos. El uso en conjunto de herramientas puede ser más útil que usar una sola. Como vimos, el diseño por computadora nos muestra una lista clara de cuáles mutaciones debemos introducir para ir hacia nuestra meta. Por otro lado, con la ingeniería de consensos aprovechamos los recursos y la diversidad que nos ofrece la naturaleza. La ingeniería de proteínas sigue avanzando para ampliar la caja de herramientas, para que podemos combinarlas y expandir nuestra capacidad para "tunear" proteínas.

\section{Referencias}

* Aerts, D., Verhaeghe, T., Joosten, H., Vriend, G. y Soetaert, W. (2013). Consensus Engineering of Sucrose Phosphorylase: The Outcome Reflects the Sequence Input. Biothechology and Bioengineering, 110(10), 2563-2572. Dol: http://doi. org/10.1002/bit.24940.

* Blau, N. (2016). Genetics of Phenylketonuria: then and now. Human mutation, 37(6), 508-515. Dol: https://doi.org/10.1002/humu.22980.

* Garcia-Guevara, F., Bravo, I., Martínez, C. y Segovia, L. (2017). Cofactor specificity switch in Shikimate dehydrogenase by rational design and consensus engineering. Protein Engineering, Design and Selection, september, 1-9. Dol: http://doi.org/10.1093/ protein/gzx031.

Kogure, T., Kubota, T., Suda, M., Hiraga, K. y Inui, M. (2016). Metabolic engineering of Corynebacterium glutamicum for shikimate overproduction by growth-arrested cell reaction. Metabolic Engineering, 38, 204-216. Dol: http://doi.org/10.1016/j. ymben.2016.08.005.

* Lehmann, M., Kostrewa, D., Wyss, M., Brugger, R., D’Arcy, A., Pasamontes, L. y van Loon, A. P. G. M. (2000). From DNA sequence to improved functionality: using protein sequence comparisons to rapidly design a thermostable consensus phytase. Protein Engineering Design and Selection, 13(1), 49-57. DOI: http://doi. org/10.1093/protein/13.1.49.

- Loening, A. M., Fenn, T. D., Wu, A. M. y Gambhir, S. S. (2006). Consensus guided mutagenesis of Renilla luciferase yields enhanced stability and light output. Protein Engineering, Design and Selection, 19(9), 391-400. Dol: http://doi.org/10.1093/ protein/gzl023. 
* Martínez, J. A., Bolívar, F. y Escalante, A. (2015). Shikimic Acid Production in Escherichia coli: From Classical Metabolic Engineering Strategies to Omics Applied to Improve Its Production. Frontiers in Bioengineering and Biotechnology, 3, 1-16. DOI: http://doi.org/10.3389/fbioe.2015.00145.

* Rane, M. J. y Calvo, K. C. (1997). Reversal of the nucleotide specificity of ketol acid reductoisomerase by site-directed mutagenesis identifies the NADPH binding site. Archives of Biochemistry and Biophysics, 338(1), 83-89. Dol: http://doi.org/10.1006/ abbi.1996.9802.

* Rodriguez, A., Martinez, J. A., Flores, N., Escalante, A., Gosset, G. y Bolivar, F. (2014). Engineering Escherichia coli to overproduce aromatic amino acids and derived compounds. Microbial Cell Factories, 13(1), 126. Dol: http://doi.org/10.1186/s12934014-0126-z.

* Scheiwiller, T. y Hirschi, S. (2010). 09 Roche Annual Report. Basel.

* Steipe, B., Schiller, B., Pluckthun, A. y Steinbacher, S. (1994). Sequence statistics reliably predict stabilizing mutations in a protein domain. Journal of Molecular Biology, 188-192. Dol: https://doi.org/10.1006/jmbi.1994.1434.

* Valero, A. y Jardón, J. (2006). ¿Qué es la evolución biológica? ¿Cómo ves?, 97, 1417 [impresa]. Disponible en: http://www.comoves.unam.mx/assets/revista/97/elagente-secreto-de-la-evolucion.pdf.

\section{Cómo citar este artículo}

* García Guevara, José Fernando (2019). "Tuneo" de proteínas: herramientas para modificarlas. Revista Digital Universitaria (RDU). Vol. 20, núm. 4 julio-agosto. Dol: http://doi.org/10.22201/codeic.16076079e.2019.v20n4.a5.

Recepción: 01/01/2018 Aprobación: 16/05/2019. 